\title{
Assessment of the effector function of CMV- specific CTLs isolated using MHC-multimers from granulocyte-colony stimulating factor mobilized peripheral blood
}

Lorea Beloki ${ }^{1}$, Miriam Ciaurriz , Cristina Mansilla' ${ }^{1}$ Amaya Zabalza' ${ }^{1}$ Estela Perez-Valderrama ${ }^{1}$, Edward R. Samuel ${ }^{2}$, Mark W. Lowdell ${ }^{2}$, Natalia Ramirez ${ }^{1^{*}+}$ and Eduardo Olavarria ${ }^{1,3 \dagger}$

\begin{abstract}
Background: Adoptive transfer of CMV-specific T cells has shown promising results in preventing pathological effects caused by opportunistic CMV infection in immunocompromised patients following allogeneic hematopoietic stem cell transplantation. The majority of studies have used steady-state leukapheresis for CMV-reactive product manufacture, a collection obtained prior to or months after G-CSF mobilization, but the procurement of this additional sample is often not available in the unrelated donor setting. If the cellular product for adoptive immunotherapy could be generated from the same G-CSF mobilized collection, the problems associated with the additional harvest could be overcome. Despite the tolerogenic effects associated with G-CSF mobilization, recent studies described that CMV-primed T cells generated from mobilized donors remain functional.
\end{abstract}

Methods: MHC-multimers are potent tools that allow the rapid production of antigen-specific CTLs. Therefore, in the present study we have assessed the feasibility and efficacy of CMV-specific CTL manufacture from G-CSF mobilized apheresis using MHC-multimers.

Results: CMV-specific CTLs can be efficiently isolated from G-CSF mobilized samples with Streptamers and are able to express activation markers and produce cytokines in response to antigenic stimulation. However, this anti-viral functionality is moderately reduced when compared to non-mobilized products.

Conclusions: The translation of Streptamer technology for the isolation of anti-viral CTLs from G-CSF mobilized PBMCs into clinical practice would widen the number of patients that could benefit from this therapeutic strategy, although our results need to be taken into consideration before the infusion of antigen-specific T cells obtained from G-CSF mobilized samples.

Keywords: Allogeneic hematopoietic stem cell transplantation, Cytomegalovirus-specific cytotoxic T cells, Streptamers, Granulocyte-colony stimulating factor, Immunotherapy

\footnotetext{
* Correspondence: nramireh@cfnavarra.es

${ }^{\dagger}$ Equal contributors

'Oncohematology Research Group, Navarrabiomed - Miguel Servet

Foundation, IDISNA (Navarra's Health Research Institute), Irunlarrea 3, 31008

Pamplona, Spain

Full list of author information is available at the end of the article
}

\section{Ciomed Central}

(c) 2015 Beloki et al. This is an Open Access article distributed under the terms of the Creative Commons Attribution License (http://creativecommons.org/licenses/by/4.0), which permits unrestricted use, distribution, and reproduction in any medium, provided the original work is properly credited. The Creative Commons Public Domain Dedication waiver (http:// creativecommons.org/publicdomain/zero/1.0/) applies to the data made available in this article, unless otherwise stated. 


\section{Background}

Allogeneic hematopoietic stem cell transplantation (alloHSCT) is usually followed by an immunosuppression period during which patients are susceptible to opportunistic infections, including human cytomegalovirus (CMV) as one of the most common viral-infections [1]. Although pharmacological treatment is routinely used prophylactically or pre-emptively to avoid reactivation or primary infection, anti-viral drugs still have some limitations since they are associated with both toxicity and delayed immune reconstitution $[2,3]$. It is widely accepted that CMV-specific cytotoxic $\mathrm{T}$ lymphocytes are essential to control virus-related complications after allo-HSCT [4]. Therefore the adoptive transfer of antigen-specific $\mathrm{T}$ cells after allo-HSCT has been investigated, showing promising results [5].

The majority of studies that generated virus-specific $\mathrm{T}$ cells have used non-mobilized peripheral blood mononuclear cells (PBMCs) isolated from an additional leukapheresis collection from the original allo-HSCT donor, different to that of the peripheral blood stem cell (PBSCs) collection performed after granulocyte-colony stimulating factor (G-CSF; Filgrastim) treatment. This additional leukapheresis harvest, apart from increasing the cost of the procedure and the discomfort caused to the donor, can be difficult to obtain in the unrelated donor setting where donor refusal or logistical and scheduling difficulties can prevent collection [6-8]. Manufacture of virus-specific $\mathrm{T}$ cells from the original G-CSF mobilized collection could potentially overcome the difficulties associated with procurement of a second apheresis.

However, G-CSF administration has been associated with a tolerogenic $\mathrm{T}$ cell phenotype; it promotes regulatory $\mathrm{T}$ cells that produce the anti-inflammatory cytokine IL-10, induces a polarization towards $\mathrm{T}$ helper cell type 2 (Th2) differentiation and while inhibiting Th cell type 1 (Th1) differentiation $[9,10]$ and decreasing the expression of genes associated with cytotoxicity, antigen presentation and graft-versus-host disease [11].

Streptamer technology avoids the strict requirements associated with advanced therapy medicinal product (ATMP) manufacture, due to minimal manipulation of direct selection [12] and holds great promise in the unrelated donor setting, widening the clinical application of this fast and simple methodology [13-15]. In this present study we evaluated the feasibility of generating therapeutic CMV-reactive cytotoxic $\mathrm{T}$ cells (CTLs) using Streptamers from G-CSF treated donor samples, and were able to show that CMV-specific $\mathrm{T}$ cells directly selected from G-CSF mobilized samples are highly functional, although their potential is slightly reduced when compared to non-mobilized CMV-specific CTLs (CMV-CTLs).

\section{Methods}

\section{Donor population and ethical statement}

This descriptive study was approved by the Institutional Review Board at Complejo Hospitalario de Navarra (CHN), and all donors gave written informed consent in accordance with the Declaration of Helsinki prior to enrolment. All samples were obtained from CMV-seropositive healthy donors who carried the HLA-A*02:01 allele. Serological analysis for CMV was obtained from the Microbiology Service of the CHN and HLA-I typing was done in the Immunology Unit of the CHN.

\section{G-CSF mobilized and non-mobilized donor sample collection and preparation}

Filgrastim treated PBMCs $(n=3)$ were obtained from leukapheresis collected from healthy donors who received $10 \mu \mathrm{g} / \mathrm{kg} /$ day of recombinant G-CSF (Sandoz Biopharmaceuticals, Paris, France) every 12 h starting five days before collection. Leukapheresis were performed with a COBE Spectra continuous flow blood cell separator (COBE Spectra apheresis system, Caridian BCT, Lakewood, CO, USA). Cell products anticoagulated with acid citrate dextrose were collected with a $1.1 \mathrm{ml} /$ min flux in a $500 \mathrm{ml}$ container, and an aliquot of $1 \mathrm{ml}$ was taken from the apheresis collection to perform the assays. Non-mobilized PBMCs $(n=3)$ were isolated from buffy coat samples anticoagulated with citrate phosphate dextrose obtained from anonymized healthy donors in the Blood and Tissue Bank of Navarra (BSTN). PBMCs were isolated by Ficoll-Paque density gradient centrifugation (GE Healthcare Bio-Sciences, Uppsala, Sweden), counted in a Neubauer Hemocytometer using $0.4 \%$ trypan blue staining (Sigma-Aldrich, St. Louis, MO) and cells were resuspended in RPMI 1640 Medium (Life Technologies, Paisley, UK) supplemented with $10 \%$ human $A B$ serum (Lonza, Basel, Switzerland). Approximately $30 \times 10^{6}$ freshly isolated PBMCs were cryopreserved for later use as feeders in functional assays. Briefly, PBMCs were mixed with fetal bovine serum (Lonza) containing $20 \%$ dimethylsulphoxide (WakChemie, Steinbach, Germany), at a 1:1 ratio.

\section{Myeloid precursor removal by plastic adherence}

In order to minimize the presence of non-specific cells in the cellular product, the sample was enriched for CTLs by a plastic adherence process [16]. In brief, $2.25 \times 10^{8}$ cells were plated in sterile $225 \mathrm{~cm}^{2} \mathrm{~A} / \mathrm{N}$ flasks with CellBIND Surface (Corning, Corning, NY) at $0.5 \times 10^{6}$ cells $/ \mathrm{ml}$ in X-VIVO 15 Serum-free cell medium w/o supplements (Lonza). Cells were incubated for $1 \mathrm{~h}$ at $37{ }^{\circ} \mathrm{C}$ and $5 \% \mathrm{CO}_{2}$. Non-adherent cells were carefully collected by aspiration to avoid the disruption of adherent myeloid cellular populations and 
were washed with Dulbecco's phosphate buffered saline (dPBS; Sigma-Aldrich) before quantification.

\section{CMV-specific CTL isolation with Streptamer technology}

Two different technical approximations were performed in order to obtain a cellular product of anti-CMV therapeutic $\mathrm{T}$ cells; in the first one, $\mathrm{CMV}$-specific $\mathrm{T}$ cells were isolated and subsequently expanded in order to obtain enough cells to perform functional assays. In the second approach, the PBMC sample was first enriched in CMVCTLs by antigen stimulation before their isolation was carried out.

\section{Isolation of CMV-specific CTLs by multimer staining and subsequent in vitro expansion}

For $50 \times 10^{6}$ non-adherent PBMCs, $3.75 \mu \mathrm{g}$ of Streptamer (ST) Magnetic Beads and $5 \mu \mathrm{g}$ of ST MHC class I (HLAA*02:01/CMVpp65-NLVPMVATV Streptamer; both from IBA GmbH, Göttigen, Germany) were incubated overnight at $4{ }^{\circ} \mathrm{C}$ in the dark to generate the ST-magnetic bead complex. This complex was added to the cell pellet and incubated for $45 \mathrm{~min}$ at $4{ }^{\circ} \mathrm{C}$ in the dark. ST+ cells were isolated using a Possel_ds selection program on the AutoMACS Pro separator (Miltenyi Biotec, Bergisch Gladbach, Germany). ST was dissociated from the eluted cells with $1 \mathrm{mM}$ D-biotin (IBA GmbH), or left bound to the cell in order to compare the effect of constant binding of the multimer to the TCR.

Following magnetic enrichment, up to $100.000 \mathrm{ST}+$ CMV-specific CTLs were co-cultured with $8 \times 10^{6} \gamma$ irradiated (30Gy) autologous PBMCs that were pre-loaded with $10 \mu \mathrm{g} / \mathrm{ml} \mathrm{CMVpp65} 5_{495-503}$ peptide (NLVPMVATV) overnight (Proimmune, Oxford, UK). The expansion was carried out in round bottom tissue culture tubes (Falcon BD Biosciences) in RPMI 1640 supplemented with $10 \%$ human $\mathrm{AB}$ serum, $1 \%$ penicillin/streptomycin (Lonza) and $10 \mathrm{ng} / \mathrm{ml}$ of IL-7 and IL-15 (Miltenyi Biotec). Cells were expanded over 21 days, culture medium was changed every 2 or 3 days and cells split when necessary. Viable cell counts were performed every 2-3 days using $0.4 \%$ trypan blue staining.

\section{CMV-specific CTL isolation after enrichment during expansion}

Before automated CMV-specific CTL selection, the number of specific $\mathrm{T}$ cells was increased by stimulating all PBMCs with the CMVpp65 $495-503$ peptide. Briefly, up to $30 \times 10^{6}$ PBMCs were cultured in round bottom culture tubes in the presence of $10 \mu \mathrm{g} / \mathrm{ml} \mathrm{CMVpp65} 5_{495-503}$ peptide and IL-7 and IL-15 as previously described, at a concentration of $5 \times 10^{5}$ cells $/ \mathrm{ml}$.

After expansion, up to $1 \times 10^{8}$ cells were stained with the ST-magnetic bead complex and ST+ cells were isolated as previously described.

\section{Characterization of specificity and immunophenotype of CMV-specific cells}

To analyze the phenotype and purity of the fresh isolated products and expanded cells, they were stained with the ST-PE complex. Briefly, $0.75 \mu \mathrm{g}$ of PE-labelled Strep-Tactin and $0.5 \mu \mathrm{g}$ of antigen-specific MHC I-Strep (HLA-A*02:01/NLVPMVATV Streptamer) were incubated during $45 \mathrm{~min}$ at $4{ }^{\circ} \mathrm{C}$ in the dark to form the STPE complex. $0.2 \mu \mathrm{g}$ of this reversible multimer were added to $1 \times 10^{6}$ cells. The incubation was carried out during $45 \mathrm{~min}$ at $4{ }^{\circ} \mathrm{C}$ in the dark and afterwards cells were stained with, CD8-FITC (BioLegend, San Diego, USA), CD3-PerCP, CD137-APC (Miltenyi Biotec), and CD4-APC-Cy7 (BD Biosciences, San Jose, USA).

During the culture period, specificity and phenotype of expanded cells were analyzed every 7 days by staining with the ST-PE complex and monoclonal antibodies as previously described, with the addition of CD69 PE-Cy7 and CD57 VioBlue (Miltenyi Biotec).

Furthermore, at the beginning and the end of the expansion the memory phenotype of the cells was analyzed by staining with CD45RA-V450 and CCR7-PE-Cy7 (both $\mathrm{BD}$ Biosciences) during $15 \mathrm{~min}$ at room temperature.

\section{Analysis of cell-surface expression of activation markers upon antigenic re-stimulation}

Expanded CMV-CTLs cells were re-stimulated with either CMVpp65 $495-503$-loaded or untouched feeders, used as CMV-stimulator or control feeders respectively, and activation marker expression was analyzed.

Briefly, autologous PBMCs were thawed out to be used as feeders and labelled with $1 \mu \mathrm{M}$ carboxyfluorescein diacetate succinimidyl ester (CFSE) to discriminate between feeders and responder CMV-CTLs during flow cytometry acquisition and analysis. Subsequently, they were plated at $3 \times 10^{6}$ cells $/ \mathrm{ml}$, and loaded with $10 \mu \mathrm{g} / \mathrm{ml}$ CMVpp65495-503 peptide to produce CMV-loaded feeders, or left untouched as control feeders and incubated overnight at $37^{\circ} \mathrm{C}$ with $5 \% \mathrm{CO}_{2}$.

Expanded CMV-CTLs were re-stimulated with either CMV-loaded or control feeders at a 2.5:1 feeder:responder ratio, with $1 \times 10^{6}$ cells/well in 96 well plates. Samples were incubated for $6 \mathrm{~h}$ and afterwards 500,000 cells were stained with anti-human CD8-PerCP, CD137-APC, CD4APC-Cy7, and CD69-PE-Cy-7 monoclonal antibodies.

\section{Evaluation of cytokine production in response to re-stimulation with the antigen}

To assess the effector activity of CMV-CTL by intracellular cytokine staining, expanded cells were co-cultured for $6 \mathrm{~h}$ with CMV-loaded or control feeders labelled with CFSE at a 2.5:1 ratio, in the same way as for surface activation marker expression analysis. The incubation was done in the presence of $1 \mu \mathrm{g} / \mathrm{ml}$ anti-CD28 (BD 
Bioscience) and $1 \mu \mathrm{g} / \mathrm{ml}$ of brefeldin A (Sigma-Aldrich). Following the incubation, $1 \times 10^{6}$ cells were stained with either PE-conjugated anti-human IFN- $\gamma$, IL-2, IL-10, TNF- $\alpha$, or Granzyme B (BD Biosciences) and with CD8PerCP and CD4-APC-Cy7 monoclonal antibodies, then fixed and permeabilized (Intrastain; DakoCytomation, Ely, UK), according to the manufacturer's instructions.

\section{Flow cytometric analysis and isotype controls}

All flow cytometry experiments consisted of five to seven color panels, where a minimum of 50.000 $\mathrm{CD} 3+$ cells were acquired. CD3+ cells were defined after gating viable lymphocyte population using the Forward Scatter (FSC) versus Side Scatter (SSC) dot-plot, and cell population percentages were derived from the $\mathrm{CD} 3+$ cell gate, except for the memory $\mathrm{T}$ cell phenotype that is derived from the $\mathrm{CD} 3+\mathrm{CD} 8+\mathrm{ST}+$ CMV-CTL gate. Data were acquired on a FACSCanto II flow cytometer (BD) and analyzed using FlowJo version 10 (TreeStar Inc., Ashland, OR, USA). Data were summarized using descriptive statistics such as mean \pm standard deviations (SD).

For cytokine and activation marker staining controls, mouse antibodies of matching isotype conjugated with $\mathrm{PE}, \mathrm{APC}$ or APC-Cy7 (BD Biosciences) were used.

\section{Results \\ CMV-CTL isolation from G-CSF mobilized and non-mobilized samples}

Streptamer technology was used to quantify and isolate CMV-CTL from non-mobilized or G-CSF mobilized PBMCs. The mean frequency of $\mathrm{CD} 3+\mathrm{CD} 8+\mathrm{ST}+$ in the G-CSF mobilized starting material was $1.56 \% \pm 2.42$, compared to $0.5 \% \pm 0.29 \mathrm{CD} 3+\mathrm{CD} 8+\mathrm{ST}+$ in nonmobilized PBMCs. It is noteworthy to mention the high variability between samples, probably due to interindividual variability because non-mobilized and G-CSF mobilized samples were collected from different individuals. CMV-CTLs were isolated from original samples using Streptamer and the purity of the obtained product was determined as the percentage of ST+ cells in the product, whereas the yield was defined as the absolute number of ST+ cells present in the positive fraction as a proportion of the absolute number of ST+ cells in the sample prior to isolation (Fig. 1a, 1b). In G-CSF mobilized samples, a mean of $20.77 \pm 7.83 \times 10^{6}$ PBMCs were used for the isolation, and a cell product made of a mean of 6.5 $\pm 3.97 \times 10^{4}$ cells with a mean purity of $75.27 \% \pm 33.15$ and yield of $56.28 \% \pm 26.14$ was obtained. From nonmobilized samples, a mean of $37.39 \pm 15.73 \times 10^{6}$ PBMCs were used to obtain a cell product made of $7.58 \pm 5.82 \times$ $10^{4}$ CMV-CTL with a mean purity of $88.9 \% \pm 13.67$ and yield of $56.19 \% \pm 33.95$.

\section{In vitro CMV-CTL expansion potential is weakened in G-CSF mobilized samples}

To increase the number of isolated CMV-specific T cells in order to perform functional assays, cells dissociated from the streptamer (Additional file 1: Figure S1) were cultured during 21 days in the presence of CMV-loaded feeders and homeostatic IL-7 and IL-15 cytokines. The proliferative ability of cells obtained from G-CSF mobilized samples or from steady state leukapheresis was evaluated (Fig. 2a). The expansion potential of CMV-CTL generated from G-CSF mobilized PBMCs revealed a mean fold expansion of $13.77 \pm$ 10.10, obtaining $1.02 \pm 0.36 \times 10^{6} \mathrm{CMV}$-CTLs after expansion. G-CSF mobilized CMV-CTL showed a reduced ability when compared to non-mobilized CMV-CTL (mean fold expansion: $263.75 \pm 192.58)$, where $25 \pm 33.69 \times 10^{6} \mathrm{CMV}-$ CTL were obtained following expansion.

\section{CMV-CTLs express the TCR that specifically recognizes the CMVpp65 ${ }_{495-503}$ peptide}

Cells were phenotypically characterized during expansion by staining with $\mathrm{T}$ cell subset markers, Streptamer, and activation markers. Before starting the expansion, the cellular products isolated from G-CSF mobilized PBMCs were predominantly CD3 + CD $8+(77.90 \% \pm$ 31.17 ), and were contaminated with a high percentage of CD3 + CD4+ $(16.00 \% \pm 22.80)$. Following 21 day culture, expanded cells showed an elevated CD3 $+\mathrm{CD} 8+\mathrm{T}$ cell population $(83.63 \% \pm 45.08)$ and a reduced $\mathrm{CD} 3+\mathrm{CD} 4+$ $\mathrm{T}$ cell population $(14.49 \% \pm 19.71)$. In comparison, streptamer isolated cells obtained from non-mobilized PBMCs, prior to culture were $92.38 \% \pm 11.88 \mathrm{CD} 3+$ $\mathrm{CD} 8+$ and $1.96 \% \pm 1.21 \mathrm{CD} 3+\mathrm{CD} 4+$. Following expansion mean proportion of $\mathrm{CD} 3+\mathrm{CD} 8+$ and $\mathrm{CD} 3+\mathrm{CD} 4+$ was $97.67 \% \pm 1.72$ and $1.04 \% \pm 1.46$, respectively. CMVpp65 $495-503$-specific TCR expression was analyzed during the expansion (Fig. 2b) and internalization of TCR complex could be observed using streptamer staining after the CMV-loaded feeder addition. The specific TCR was re-expressed from day 7 and expression was maintained until the end of the culture period. After 21 day expansion, the proportion of $\mathrm{CD} 3+\mathrm{CD} 8+\mathrm{ST}+$ in G-CSF mobilized cells was $75.4 \% \pm 26.69$, and in nonmobilized CMV-CTLs the frequency was $98.17 \% \pm 2.34$.

CD137 and CD69 activation marker expression was analyzed throughout the 21 day culture period (Fig. 2c); CD137 expression was undetectable from the beginning of the expansion and CD69 was heterogeneously and unpredictably changed during the culture, for both GCSF mobilized and non-mobilized samples.

\section{G-CSF mobilized CMV-CTL evolve towards a terminally differentiated phenotype during culture}

Senescence of expanded cells was investigated during the culture period by CD57 expression analysis (Fig. 3a) 


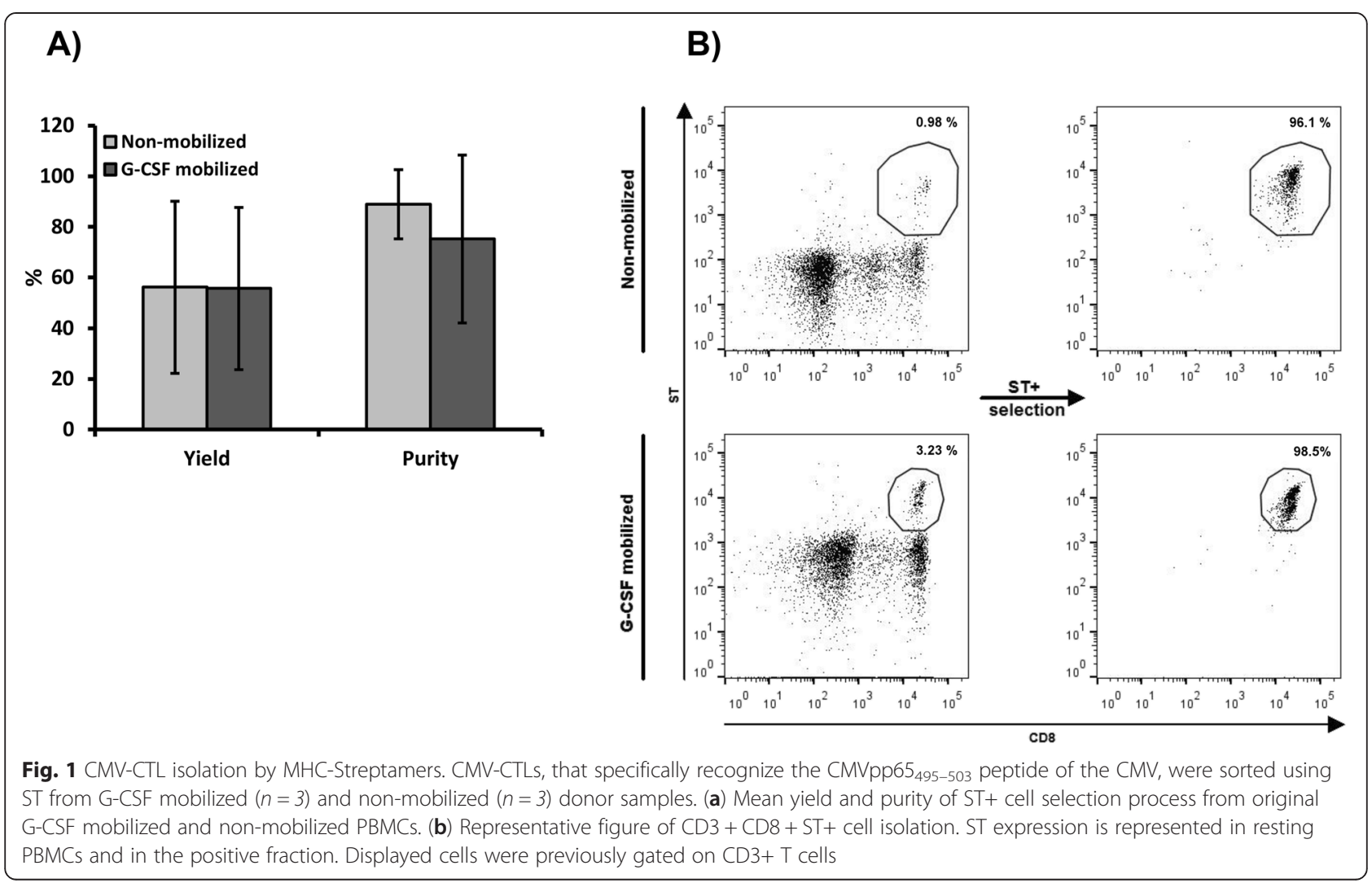

and showed an increase during the 21 day culture period. At the beginning of the expansion, mean CD57 expression was $9.72 \% \pm 7.75$ and $9.50 \% \pm 10.85$ in G-CSF mobilized and non-mobilized CMV-CTL, respectively. In comparison, after culture mean CD57 expression increased to $52.06 \% \pm 27.52$ in the mobilized CMV-CTL product, and to $81.15 \% \pm 3.94$ in non-mobilized samples.

Memory phenotype of CMV-CTL before isolation, in the positive fraction of ST+ cell products and after expansion was analyzed by staining with CD45RA and CCR7 markers (Fig. 3b, c), and memory subpopulations defined as follows: naïve $T$ cells $\left(T_{N}, C D 45 R A+C C R 7+\right)$, central memory $\mathrm{T}$ cells $\left(\mathrm{T}_{\mathrm{CM}}, \mathrm{CD} 45 \mathrm{RA}-\mathrm{CCR} 7+\right)$, effector memory $\mathrm{T}$ cells ( $\left.\mathrm{T}_{\mathrm{EM}}, \mathrm{CD} 45 \mathrm{RA}-\mathrm{CCR} 7-\right)$, and terminally differentiated effector memory $\mathrm{T}$ cells ( $\mathrm{T}_{\mathrm{EMRA}}$, CD45RA + CCR7-). CMV-CTL obtained from both G-CSF mobilized and nonmobilized PBMCs were predominantly $\mathrm{T}_{\mathrm{EM}}$ with a considerable $\mathrm{T}_{\text {EMRA }}$ population (Table 1). Memory phenotype was not notably modified when comparing cells before and after isolation or at the end of the expansion period.

\section{Expanded CMV-CTL express activation markers and produce pro-inflammatory cytokines and granzyme B} After culture, the effector functional capacity of expanded CMV-CTL was assessed by quantifying activation marker expression and cytokine production upon re-stimulation with the CMVpp65 $495-503$ peptide.
In G-CSF mobilized CMV-CTL, $73.05 \% \pm 17.61$ cells re-expressed CD137 activation marker after antigenic rechallenge, and $90.50 \% \pm 12.16$ were $\mathrm{CD} 69+$. These values were similar to the expression observed in non-mobilized CMV-CTLs (CD137+, $67.67 \% \pm 8.10$; CD69+, $93.53 \% \pm$ 5.58) (Fig. 4a, b).

Intracellular cytokine staining following antigenic rechallenge illustrated that CMV-CTL generated from both mobilized and non-mobilized PBMCs were able to secrete pro-inflammatory cytokines and granzyme B (Fig. 4c, d). The secretion cytokine pattern in G-CSF mobilized and non-mobilized CMV-CTLs was similar; G-CSF mobilized CMV-CTLs produced high levels of IFN- $\gamma(82.10 \% \pm 19.86)$ and granzyme B (53.67 \% \pm 21.83). A lower percentage produced TNF- $\alpha(7.62 \% \pm$ $1.05)$ and IL-2 (1.44 $\% \pm 0.84)$, with undetectable levels of IL-10 anti-inflammatory cytokine production. However, the frequency of cytokine secreting cells was slightly reduced when compared to that observed in non-mobilized cells $(96.27 \% \pm 1.57$ IFN- $\gamma+, 79.30 \% \pm$ 22.00 granzyme $\mathrm{B}+, 38.83 \% \pm 14.06 \mathrm{TNF}-\alpha+, 4.84 \% \pm$ $1.75 \mathrm{IL}-2+$ and undetectable levels of IL-10).

Functional CMV-CTL can be manufactured by Streptamer isolation after previous antigen-specific cell enrichment A different approach for CMV-specific $\mathrm{T}$ cell product generation was accordingly assessed, from both mobilized 


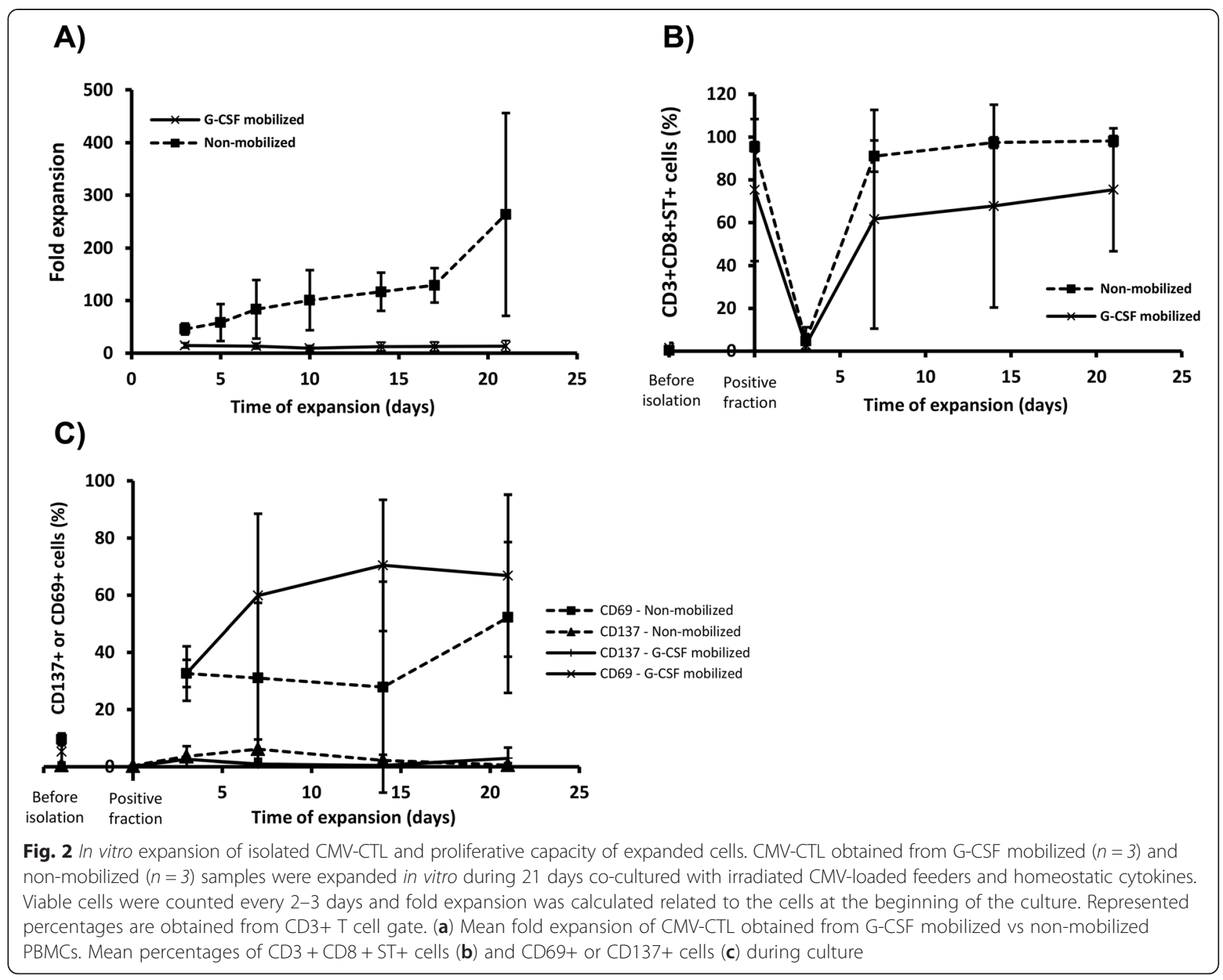

and non-mobilized samples, by first enriching CMV-CTL percentage in the original sample. PBMCs were stimulated with CMVpp65 $495-503$ antigen to stimulate CMV-CTL proliferation, expanded in the presence of IL-7 and IL-15, and CMV-CTLs were finally isolated using ST. The function of the obtained cellular product was compared to isolated directly from original PBMCs.

Following antigen-specific stimulation of G-CSF mobilized PBMCs, CMV-CTL percentage increased from 1.62 $\% \pm 2.42$ at the beginning to $60.23 \% \pm 48.87$ after 21 day culture, with a mean fold increase of CMV-CTL subpopulation of 361.85 (range 23.41 - 791.03). On the other hand, CMV-CTL percentage in non-mobilized PBMCs increased from $0.20 \% \pm 0.19$ to $18.62 \% \pm 13.35$ during culture, and the absolute CMV-CTL number raised by 451.08 fold (range 7.73 - 794.73), similar to G-CSF mobilized CMV-CTLs (Fig. 5a). CMV-specific T cell isolation after expansion of G-CSF mobilized PBMCs showed a mean purity and yield of $84.4 \% \pm 25.39$ and $24.98 \% \pm$ 20.80, respectively. CMV-specific $\mathrm{T}$ cell isolation from non-mobilized samples showed similar results, with a mean purity of $98.67 \% \pm 0.25$ and yield of $36.39 \% \pm$ 14.79 (Fig. 5b). Yield was slightly reduced when compared to direct isolation of CMV-CTL from the original PBMC samples.

In G-CSF mobilized samples, CD57 expression was $19.77 \% \pm 19.19$ in original PBMCs, it was increased to $49.78 \% \pm 26.58$ CD $57+$ by the end of the expansion, and CVM-CTL isolated after culture were $53.57 \% \pm 29.75$ CD57+. On the other hand, when non-mobilized samples were used, $17.22 \% \pm 9.90 \mathrm{CD} 57+$ were present in the original samples and the expression was increased to $67.31 \% \pm 6.07$ at the end of the expansion, and after isolation the CMV-CTL product expressed $72.47 \% \pm 19.49$ CD57+. CD57 expression in the CMV-CTL product was higher when compared to CMV-specific cells isolated with ST directly from the original PBMCs, for both GCSF mobilized (9.72 \% \pm 7.75 CD57+) and non-mobilized samples $(9.50 \% \pm 10.85)$. However, when directly isolated CMV-CTL were afterwards expanded during 21 days, the differences were lost (G-CSF mobilized CMV-CTL, $52.06 \% \pm 27.52 \mathrm{CD} 57+$; non-mobilized CMV-CTL, 


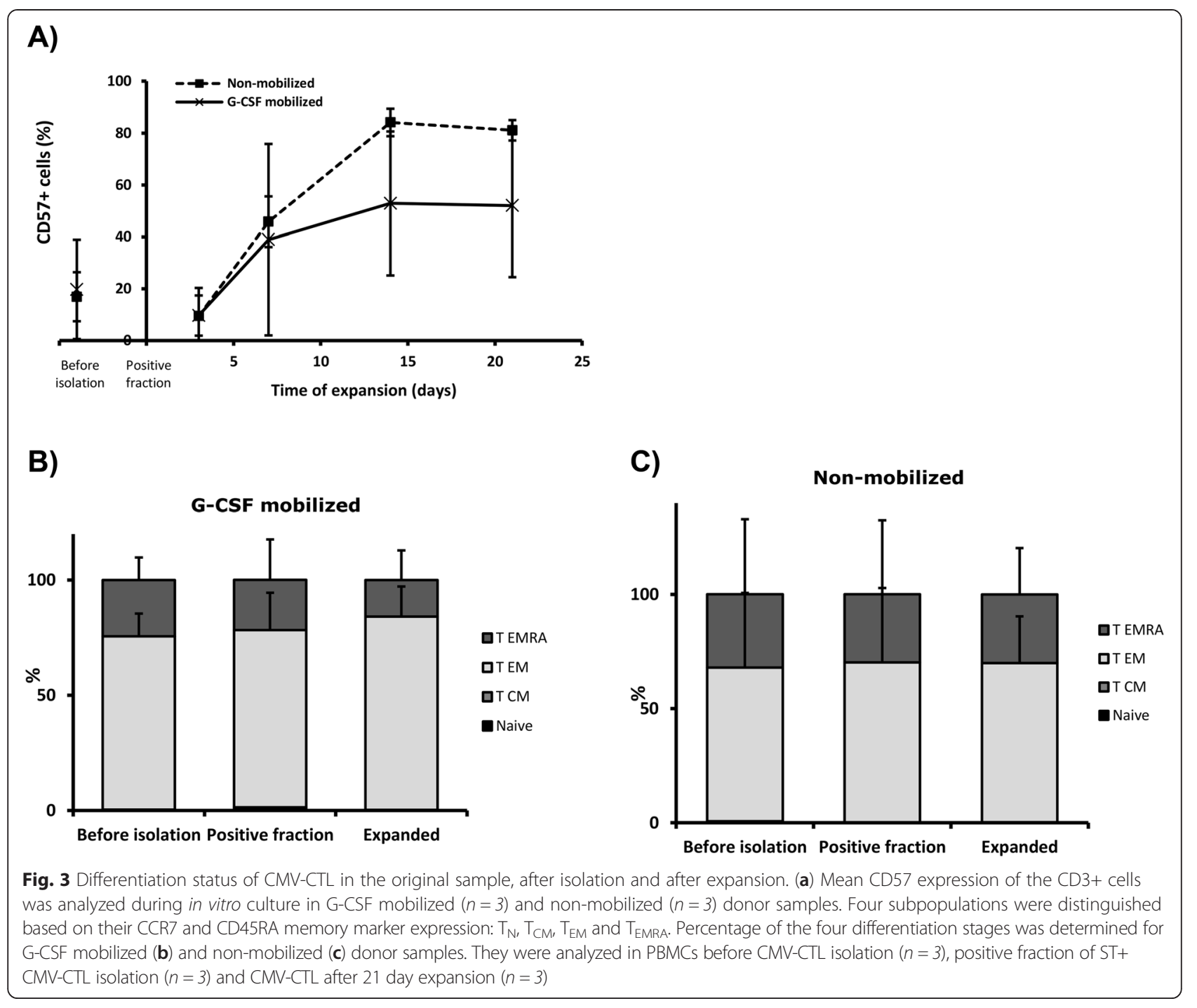

$81.15 \% \pm 3.94$ CD57+). Memory marker expression showed that, in both G-CSF mobilized (Fig. 5c) and non-mobilized (Fig. 5d) donor samples, during culture CMV-CTL $\mathrm{T}_{\text {EMRA }}$ population decrease, that could denote the poor proliferative capacity and senescence of latedifferentiated cells. Therefore, the CMV-CTL product obtained was mainly formed of $\mathrm{T}_{\mathrm{EM}}$ cells (Table 2).

The functionality of cells isolated after antigen stimulation and culture was similar to the product obtained by direct selection and expansion (Table 3); after restimulation with the antigen they re-expressed CD137 and CD69 activation markers (Fig. 5e) and produced high levels of IFN- $\gamma$ and granzyme $\mathrm{B}$, lower levels of TNF- $\alpha$ and IL-2, and undetectable levels of IL-10 (Fig. 5f). It is important to mention that TNF- $\alpha$ and granzyme B production by CMV-CTL isolated from GCSF mobilized samples is partly reduced when compared to non-mobilized CMV-CTLs.

\section{Discussion}

Adoptive transfer of CMV-specific T cell products generated from the original PBSC donor has shown promising results in the field of cellular therapy [5, 17]. Most protocols have used non-mobilized apheresis samples as starting material, but the manufacture of the therapeutic product from the same G-CSF mobilized sample used for PBSC collection offers great logistical and regulatory advantages that could avoid the need for successive blood donations and consequently widen the applicability of the adoptive immunotherapy. Recently, the feasibility of CMV-specific $\mathrm{T}$ cell generation from a G-CSF mobilized collection has been assessed, confirming the applicability of the procedure $[6-8,18]$. Despite these new findings, there is a concern about the effectiveness of the generated cell products due to the immunosuppressive effects associated with G-CSF administration $[9,19,20]$. Furthermore, it has been described that the 
Table 1 Differentiation status of directly selected CMV-CTL

\begin{tabular}{|c|c|c|c|c|c|c|c|c|}
\hline & \multicolumn{4}{|c|}{ G-CSF mobilized } & \multicolumn{4}{|c|}{ Non-mobilized } \\
\hline & $\mathrm{T}_{\mathrm{N}}$ & $T_{C M}$ & $T_{E M}$ & $\mathrm{~T}_{\text {EMRA }}$ & $\overline{T_{N}}$ & $T_{C M}$ & $T_{E M}$ & $\mathrm{~T}_{\text {EMRA }}$ \\
\hline Before isolation & $0.35 \% \pm 0.07$ & $0.05 \% \pm 0.07$ & $75.10 \% \pm 9.90$ & $24.50 \% \pm 9.76$ & $0.50 \% \pm 0.87$ & $0.13 \% \pm 0.23$ & $67.33 \% \pm 32.66$ & $32.07 \% \pm 32.98$ \\
\hline Positive Fraction & $0.50 \% \pm 0.40$ & $0.93 \% \pm 1.45$ & $76.77 \% \pm 16.26$ & $21.87 \% \pm 17.56$ & $0.00 \% \pm 0.00$ & $0.03 \% \pm 0.06$ & $70.27 \% \pm 32.51$ & $29.73 \% \pm 32.51$ \\
\hline Expanded CMV-CTL & $0.30 \% \pm 0.30$ & $0.00 \% \pm 0.00$ & $83.8 \% \pm 13.07$ & $15.90 \% \pm 12.86$ & $0.13 \% \pm 0.12$ & $0.07 \% \pm 0.12$ & $69.77 \% \pm 20.40$ & $30.03 \% \pm 20.39$ \\
\hline
\end{tabular}

$\mathrm{T}_{\mathrm{N}}, \mathrm{T}_{\mathrm{CM}}, \mathrm{T}_{\mathrm{EM}}$ and $\mathrm{T}_{\mathrm{EMRA}}$ subpopulations were determined for G-CSF mobilized and non-mobilized samples in PBMCs before CMV-CTL isolation ( $n=3$ ), positive fraction of ST+ CMV-CTL isolation $(n=3)$, and CMV-CTL after 21 day in vitro expansion $(n=3)$. Percentages were obtained from the CD3 + CD8 + ST+ cell subpopulation

background staining of MHC-multimers using G-CSF mobilized samples is higher compared to non-mobilized sample staining [20], which would negatively affect the isolation of CMV-CTLs. However, there are currently no studies that evaluate virus-specific $\mathrm{T}$ cell production from G-CSF mobilized samples by direct isolation with MHC-multimers. In this study we have investigated whether antigen-specific $\mathrm{T}$ cells can be efficiently isolated from G-CSF mobilized samples using reversible Streptamer technology and if $\mathrm{T}$ cell products manufactured from mobilized PBMCs with this technology are functionally competent and comparable to those obtained from non-mobilized donors.
After direct CMV-specific T cell isolation from G-CSF mobilized and non-mobilized samples using Streptamers, CMV-CTLs were expanded in culture in order to obtain sufficient cell numbers to perform functional assays. This three week culture could reduce the negative effect of GCSF or the effect of G-CSF might be abrogated during the expansion, but it was the only option we had to perform functional assessment of isolated CMV-CTLs. CMVCTLs were dissociated from Streptamers and expanded by co-culture with CMV-loaded autologous feeders and homeostatic cytokines. Feeders were loaded with the CMVpp65495-503 peptide since our group has previously described that isolated cells are not able to persist in vitro

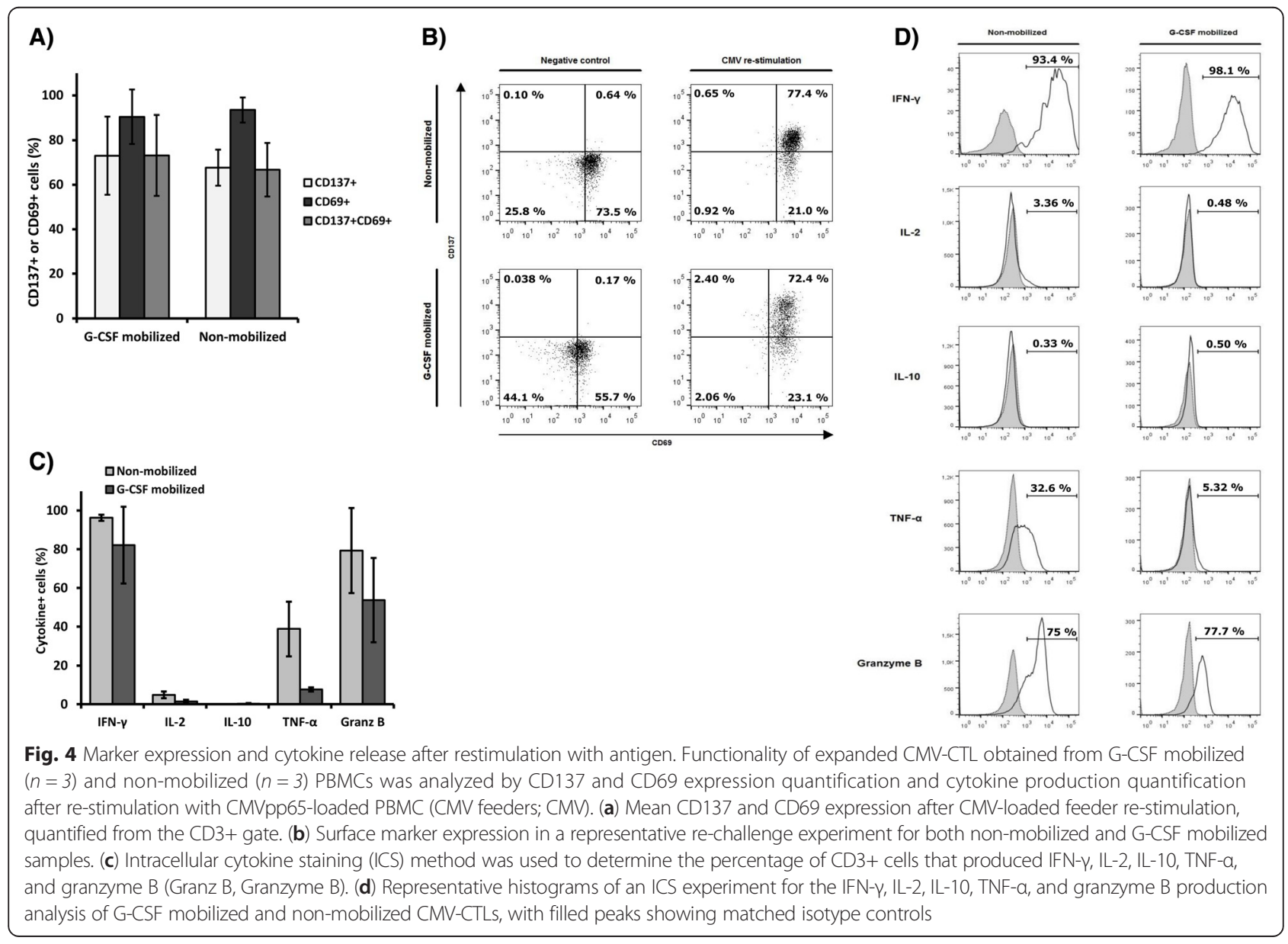




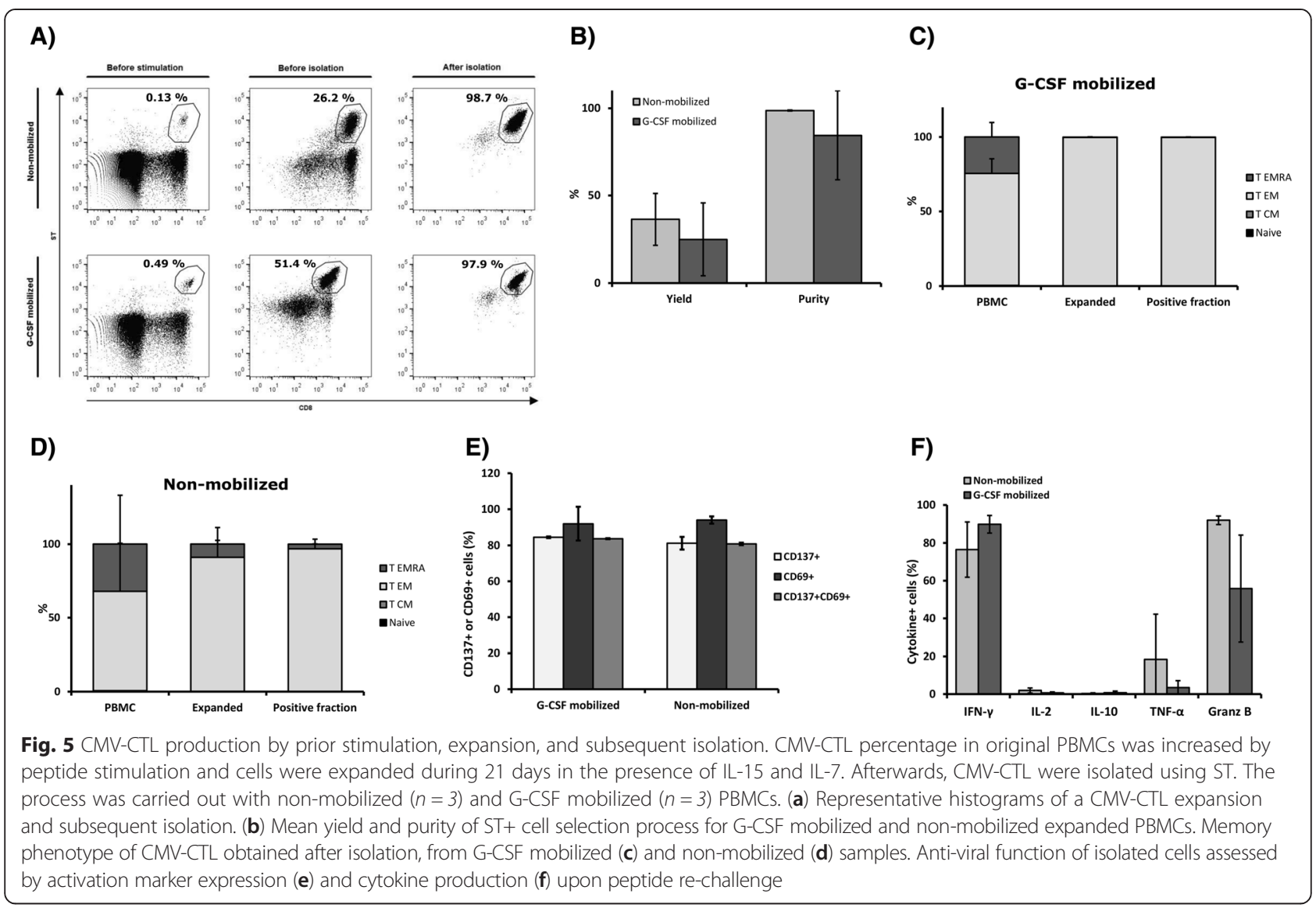

if they are not stimulated with the antigen [unpublished data]. Accordingly, IL-7 and IL-15 were used due to their importance for $\mathrm{T}$ cell homeostatic proliferation and survival [21, 22].

Although purity and yield of CMV-CTL isolation from G-CSF mobilized donors was comparable to non-mobilized PBMCs, the proliferative potential of cells obtained from G-CSF mobilized PBMCs appeared to be reduced when compared to non-mobilized samples when selected cells were expanded in vitro. A possible explanation could be that the tolerogenic effect induced by G-CSF administration could negatively affect the proliferation of CMV-CTLs in vitro. However, when all PBMCs were stimulated with the antigen in order to induce CMV-CTL enrichment before isolation, the expansion potential of CMV-specific cells in G-CSF mobilized samples was comparable to nonmobilized ones. We hypothesize that potential inadequacies in the in vitro proliferation protocol resulted in reduced proliferation of G-CSF mobilized CMV-CTLs and that different culture conditions may improve proliferation. Furthermore, in our previous studies where CMV-specific T cells were isolated through CD25, CD137, or CD154 activation marker expression and expanded in the same conditions described here $[6,8,18]$, cells isolated from G-CSF mobilized PBMCs were able to proliferate as efficiently as non-mobilized $\mathrm{CMV}$-specific $\mathrm{T}$ cells. In these cases, the

Table 2 Differentiation status CMV-CTL isolated after enrichment by three week expansion in the presence of CMVpp65495-503 peptide

\begin{tabular}{|c|c|c|c|c|c|c|c|c|}
\hline & \multicolumn{4}{|c|}{ G-CSF mobilized } & \multicolumn{4}{|c|}{ Non-mobilized } \\
\hline & $T_{N}$ & $T_{C M}$ & $T_{E M}$ & $T_{\text {EMRA }}$ & $T_{N}$ & $T_{C M}$ & $T_{E M}$ & $T_{\text {EMRA }}$ \\
\hline Before expansion & $0.35 \% \pm 0.07$ & $0.05 \% \pm 0.07$ & $75.10 \% \pm 9.90$ & $24.50 \% \pm 9.76$ & $0.50 \% \pm 0.87$ & $0.13 \% \pm 0.23$ & $67.33 \% \pm 32.66$ & $32.07 \% \pm 32.98$ \\
\hline Expanded PBMCs & $0.05 \% \pm 0.07$ & $0.15 \% \pm 0.07$ & $99.55 \% \pm 0.07$ & $0.20 \% \pm 0.14$ & $0.17 \% \pm 0.29$ & $0.03 \% \pm 0.06$ & $90.87 \% \pm 11.52$ & 8.979 \\
\hline Positive fraction & $0.00 \% \pm 0.00$ & $0.10 \% \pm 0.14$ & $99.70 \% \pm 0.14$ & $0.15 \% \pm 0.07$ & $0.00 \% \pm 0.00$ & $0.10 \% \pm 0.10$ & $96.70 \% \pm 3.32$ & $3.20 \% \pm 3.39$ \\
\hline
\end{tabular}

$\mathrm{T}_{\mathrm{N}}, \mathrm{T}_{\mathrm{CM}}, \mathrm{T}_{\mathrm{EM}}$ and $\mathrm{T}_{\mathrm{EMRA}}$ subpopulations were determined for G-CSF mobilized and non-mobilized samples in the original PBMC sample ( $n=3$ ), PBMCs after 21 day in vitro expansion $(n=3)$, and positive fraction of CMV-CTL isolation after culture $(n=3)$. Percentages were obtained from the CD3 + CD8 + ST+ cell subpopulation 
Table 3 Functional activity of CMV-CTL isolated after three week expansion

\begin{tabular}{lcc}
\hline & G-CSF mobilized & Non-mobilized \\
\hline CD137+ & \multicolumn{1}{c}{ Activation marker expression } \\
CD69+ & $84.50 \% \pm 0.42$ & $81.20 \% \pm 3.54$ \\
CD137+CD69+ & $92.00 \% \pm 9.33$ & $94.10 \% \pm 1.98$ \\
& $83.70 \% \pm 0.28$ & $80.80 \% \pm 0.71$ \\
IFN-y & \multicolumn{2}{c}{ Cytokine production } \\
IL-2 & $89.93 \% \pm 4.65$ & $76.50 \% \pm 14.64$ \\
IL-10 & $0.61 \% \pm 0.56$ & $1.91 \% \pm 1.30$ \\
TNF- $a$ & $0.71 \% \pm 0.84$ & $0.27 \% \pm 0.41$ \\
Granzyme B & $3.43 \% \pm 3.69$ & $18.42 \% \pm 23.85$ \\
\hline Acivton maker & $55.83 \% \pm 28.36$ & $92.07 \% \pm 2.28$ \\
\hline
\end{tabular}

Activation marker re-expression and cytokine production in response to antigenic re-challenge was assessed in CMV-CTLs obtained from G-CSF mobilized $(n=3)$ and non-mobilized $(n=3)$ PBMCs after 21 day expansion in the presence of the peptide. Percentages were obtained from the $\mathrm{CD} 3+$ cell gate

cellular products were made of $\mathrm{CD} 4+$ and $\mathrm{CD} 8+$ cell subsets. Therefore, the impaired expansion potential described in this study could denote that G-CSF immunosuppression negatively affects CMV-specific CTL but not helper $\mathrm{T}$ cell proliferation, or that isolated antigen-specific CD8+ cells need CD4+ help in order to overcome the immunosuppression exerted by G-CSF, that could be related to the importance of CD4+ T cells in the maintenance of a functional memory CD8+ T cell pool [23]. Nevertheless, CMV-CTLs directly isolated from G-CSF mobilized donor PBMCs may be able to expand more efficiently in vivo, since the lymphopenic environment associated with HSCT is likely to be favorable for $\mathrm{T}$ cell proliferation [24, 25], and could help in overcoming the problems associated with our proposed in vitro expansion protocol.

Differentiation status of CMV-CTLs was also assessed during expansion. CD57 expression was analyzed, a marker of functional immune senescence of $\mathrm{T}$ cells that is associated with an impaired proliferation and survival $[26,27]$. It could be determined that CD57 expression is increased in both non-mobilized and mobilized samples during expansion, reflecting that CMV-CTL undergo several rounds of cell divisions. Since it has been shown that both proliferative capacity and survival of terminally differentiated CD8 + CD57+ donor T cells are limited following their transfer to HSCT recipients [28], these data suggest that expanded CMVCTLs cells would potentially persist less in the recipient compared to cells selected and directly infused into the patient. By the end of the expansion, CD57 expression in non-mobilized samples was higher than G-CSF mobilized CMV-CTL, which correlates with the higher fold expansion of non-mobilized CMV-CTLs observed during culture.
The differentiation status of the cells was further characterized by analyzing the immunological phenotype of CMV-CTLs using CD45RA and CCR7 memory markers to distinguish between $\mathrm{T}_{\mathrm{N}}, \mathrm{T}_{\mathrm{CM}}, \mathrm{T}_{\mathrm{EM}}$, and $\mathrm{T}_{\mathrm{EMRA}}$ subpopulations [29]. CMV-CTL products, from both G-CSF mobilized and non-mobilized samples were mainly composed of $\mathrm{T}_{\mathrm{EM}}$ cells and a noteworthy proportion of $\mathrm{T}_{\mathrm{EMRA}}$ cells, during all the studied stages. These results correlate with the CD57 expression that characterizes $\mathrm{T}_{\mathrm{EMRA}}$ cells and as well as proportion of $\mathrm{T}_{\mathrm{EM}}$ cells $[30,31]$. These $\mathrm{T}_{\mathrm{EM}}$ and $\mathrm{T}_{\mathrm{EMRA}}$ subpopulations which accordingly express CD57+ maintain their proinflammatory cytokine production capacity as well as high cytotoxic effector properties, thereby conferring effective immediate protection [32-34].

After expansion, specificity and effector function of CMV-CTL obtained from both sample sources was analyzed following antigenic re-challenge. The vast majority of cells in the cellular products expressed the TCR recognizing the CMVpp65495-503 peptide demonstrating the high specificity of the product. At the beginning of the expansion TCR suffered a downregulation following the activation, which is known to be correlated with antigen sensitivity [35]. A notable proportion of cells expressed CD69 at the end of the expansion, which could be related to the IL- 15 present in the medium [36], and upon antigen-specific stimulation a high proportion of CD69+ cells accordingly re-expressed CD137.

Regarding cytokine production upon antigenic restimulation, the majority of expanded CMV-CTL from both G-CSF mobilized and non-mobilized samples released the pro-inflammatory cytokine IFN- $\gamma$, and lower levels produced TNF- $\alpha$ and IL-2. No IL-10 secretion was observed. This type 1 cytokine secretion profile illustrates the anti-viral functionality of CMV-CTL generated in our protocol [35]. They also produced high levels of the lytic molecule granzyme $\mathrm{B}$, even prior to antigenic re-stimulation, showing their cytotoxic ability since it has been described how the preformation of mature secretory lysosomes containing lytic proteins correlates with the killing ability of cytotoxic cells [37]. This cytokine secretion pattern and granzyme B formation, together with their ability to express activation markers such as CD137 and CD69, illustrates their ability to rapidly activate and induce an effective response after encountering the antigen. Furthermore, it reveals their capacity to confer an immediate effector function, that correlates with the described effector memory $\mathrm{T}$ cell phenotype [38].

For the clinical translation of this procedure, we would first eliminate myeloid progenitors of G-CSF mobilized samples performing the plastic adherent process, and subsequently obtained cells would be frozen for storage. When CMV-CTLs were needed, the cells would be thawed 
and specific cells would be isolated using Streptamer technology, since it has been previously described that CMVspecific $\mathrm{T}$ cells can be isolated from frozen cells using MHC-multimers and are totally functional when administered in vivo [39].

Finally, we also analyzed an alternative method for the generation of CMV-CTLs. In cases where detectable CMV-CTL in the G-CSF mobilized sample is limited and the purity of the obtained cell product could potentially be reduced [40], CMV-CTL could be enriched in the original sample by antigen-specific stimulation before isolation with the MHC-multimer. The cellular product obtained with this alternative method maintained their functional activity and specificity, although TNF- $\alpha$ and granzyme B production by G-CSF mobilized CMV-CTL was slightly reduced if compared to nonmobilized cells. CD57 expression in CMV-CTL increases during culture, that could potentially impair their longterm maintenance in vivo [28]. Furthermore, the cellular product manufactured following antigenic stimulation prior to direct selection would be considered an ATMP with impact on associated regulatory issues and increased costs. Therefore, even though this method could be helpful for the isolation from samples with low antigen-specific cell frequencies, our results do not support it implementation.

\section{Conclusions}

Anti-viral product manufacture from the original G-CSF mobilized PBSC graft offers great advantages mainly in the unrelated donor setting, increasing the number of patients that can benefit from this treatment. This, together with the straightforward CMV-specific $\mathrm{T}$ cell production using GMP-grade Streptamers, enhances the broad application into clinical routine of this therapy.

In this study we have been able to manufacture CMVspecific CTLs from G-CSF mobilized donor samples using Streptamer technology that present their anti-viral function, determined by their high specificity and fast and efficient response upon antigen-specific stimulation. However, the capacity to generate pro-inflammatory cytokines and lytic molecules was slightly reduced and their proliferative potential was affected when compared to non-mobilized cell products. Thus, whether the adoptive transfer of a CMV-CTL product from G-CSF mobilized PBMCs can efficiently respond in vivo and control CMV disease must be answered in future animal studies and clinical trials.

\section{Additional file}

Additional file 1: Figure S1. Expansion and functionality of CMV-CTL with or without ST dissociation. ST was dissociated $(n=3)$ or left untouched $(n=3)$ from CMV-CTL isolated from non-mobilized samples, and cells were expanded and functionally characterized afterwards. (A) Mean fold expansion of CMV-CTL obtained from non-mobilized samples, with and without ST dissociation using D-biotin. Activation marker expression (B) and cytokine production (C) upon antigenic re-stimulation in expanded CMV-CTL with ST bound to the cell surface or ST dissociated from the TCR.

\section{Abbreviations}

Allo-HSCT: Allogeneic hematopoietic stem cell transplantation; ATMP: Advanced therapy medicinal product; CMV: Cytomegalovirus; CMV-CTL: Cytomegalovirus-specific cytotoxic T cell; CTL: Cytotoxic T cell; G-CSF: Granulocyte-colony stimulating factor; PBMC: Peripheral blood mononuclear cells; PBSC: Peripheral blood stem cel; ST: Streptamer; Th: Helper T cell.

\section{Competing interests}

MWL is a shareholder in Cell Medica, a clinical-stage cellular therapeutics company. The authors have declared that there are no financial conflicts of interest in regard to this work.

\section{Authors' contributions}

$L B, N R$ and EO conceived and designed the experiments. LB performed the experiments. $L B, M C, C M, A Z, E P V$ processed and analysed the data. $L B$ wrote the manuscript with NR and EO. MWL and ERS contributed to the drafting of the manuscript. All authors read and approved the final manuscript.

\section{Acknowledgements}

This work was supported by a research grant (PI10/00136) from Fondo de Investigaciones Sanitarias (FIS) granted by the Instituto de Salud Carlos III (ISCIII). LB is a recipient of APPICS Predoctoral Fellowship from Departamento de Salud del Gobierno de Navarra. MC is a recipient of PFIS Predoctoral Fellowship from ISCIII. CM is a recipient of ANABASID Postdoctoral Fellowship from Departamento de Educación del Gobierno de Navarra. AZ is a recipient of a Post-MIR Fellowship from Complejo Hospitalario de Navarra $(\mathrm{CHN})$. We thank the staff of the Department of Haematology from Complejo Hospitalario de Navarra and of the Blood and Tissue Bank of Navarra for provision of donor material.

\section{Author details}

${ }^{1}$ Oncohematology Research Group, Navarrabiomed - Miguel Servet Foundation, IDISNA (Navarra's Health Research Institute), Irunlarrea 3, 31008 Pamplona, Spain. ${ }^{2}$ Department of Haematology, University College London Medical School, University College London, London, UK. ${ }^{3}$ Department of Haematology, Complejo Hospitalario de Navarra, Navarra Health Service, IDISNA (Navarra's Health Research Institute), Pamplona, Spain.

Received: 15 February 2015 Accepted: 4 May 2015

Published online: 20 May 2015

\section{References}

1. Busca A. Cytomegalovirus (CMV) infection after hematopoietic stem cell transplantation: significant progress, but many unresolved problems. Expert Opin Biol Ther. 2009;9:383-5.

2. Li CR, Greenberg PD, Gilbert MJ, Goodrich JM, Riddell SR. Recovery of HLA-restricted cytomegalovirus (CMV)-specific T-cell responses after allogeneic bone marrow transplant: correlation with CMV disease and effect of ganciclovir prophylaxis. Blood. 1994;83:1971-9.

3. Battiwalla M, Wu Y, Bajwa RP, Radovic M, Almyroudis NG, Segal BH, et al. Ganciclovir inhibits lymphocyte proliferation by impairing DNA synthesis. Biol Blood Marrow Transplant. 2007;13:765-70.

4. Tormo N, Solano C, Benet I, Nieto J, de la Camara R, Lopez J, et al. Reconstitution of CMV pp 65 and IE-1-specific IFN-gamma CD8(+) and CD4(+) T-cell responses affording protection from CMV DNAemia following allogeneic hematopoietic SCT. Bone Marrow Transplant. 2011;46:1437-43.

5. Ramirez N, Beloki L, Ciaurriz M, Rodriguez-Calvillo M, Escors D, Mansilla C, et al. Impact of T cell selection methods in the success of clinical adoptive immunotherapy. Cell Mol Life Sci. 2013;71:1211-24.

6. Samuel ER, Newton K, Mackinnon S, Lowdell MW. Successful isolation and expansion of CMV-reactive T cells from G-CSF mobilized donors that retain a strong cytotoxic effector function. Br J Haematol. 2013;160:87-100. 
7. Clancy LE, Blyth E, Simms R, Micklethwaite KP, Kris Ma CK, Burgess J, et al. CMV-specific cytotoxic T lymphocytes can be efficiently expanded from G-CSF mobilised haemopoietic progenitor cell products ex vivo and safely transferred to stem cell transplant recipients to facilitate immune reconstitution. Biol Blood Marrow Transplant. 2013, doi: 10.1016/j.bbmt.2013.01.021.

8. Samuel ER, Beloki L, Newton K, Mackinnon S, Lowdell MW. Isolation of highly suppressive $\mathrm{cd} 25+$ foxp $3+\mathrm{t}$ regulatory cells from $\mathrm{g}$-csf-mobilized donors with retention of cytotoxic anti-viral ctls: Application for multi-functional immunotherapy post stem cell transplantation. PLoS One. 2014;9:e85911.

9. Rutella S, Zavala F, Danese S, Kared H, Leone G. Granulocyte colony-stimulating factor: a novel mediator of T cell tolerance. J Immunol. 2005;175:7085-91.

10. Rossetti M, Gregori S, Roncarolo MG. Granulocyte-colony stimulating factor drives the in vitro differentiation of human dendritic cells that induce anergy in naive T cells. Eur J Immunol. 2010;40:3097-106.

11. Toh HC, Sun L, Soe Y, Wu Y, Phoon YP, Chia WK, et al. G-CSF induces a potentially tolerant gene and immunophenotype profile in T cells in vivo. Clin Immunol. 2009:132:83-92.

12. Neudorfer J, Schmidt B, Huster KM, Anderl F, Schiemann M, Holzapfel G, et al. Reversible HLA multimers (Streptamers) for the isolation of human cytotoxic T lymphocytes functionally active against tumor- and virus-derived antigens. J Immunol Methods. 2007;320:119-31.

13. Schmitt A, Tonn T, Busch DH, Grigoleit GU, Einsele H, Odendahl M, et al. Adoptive transfer and selective reconstitution of streptamer-selected cytomegalovirus-specific CD8+ T cells leads to virus clearance in patients after allogeneic peripheral blood stem cell transplantation. Transfusion. 2010;51:591-9.

14. Stemberger C, Graef P, Odendahl M, Albrecht J, Dossinger G, Anderl F, et al. Lowest numbers of primary CD8(+) T cells can reconstitute protective immunity upon adoptive immunotherapy. Blood. 2014;124:628-37.

15. Odendahl M, Grigoleit GU, Bonig H, Neuenhahn M, Albrecht J, Anderl F, et al. Clinical-scale isolation of 'minimally manipulated' cytomegalovirus-specific donor lymphocytes for the treatment of refractory cytomegalovirus disease. Cytotherapy. 2014;16:1245-56.

16. Beloki L, Ciaurriz M, Mansilla C, Zabalza A, Perez-Valderrama E, Samuel ER, et al. CMV-specific T cell isolation from G-CSF mobilized Peripheral Blood: depletion of myeloid progenitors eliminates non-specific binding of MHC-multimers. J Transl Med. 2014;19(12):317.

17. Saglio F, Hanley PJ, Bollard CM. The time is now: moving toward virus-specific T cells after allogeneic hematopoietic stem cell transplantation as the standard of care. Cytotherapy. 2014;16:149-59.

18. Beloki L, Ramirez N, Olavarria E, Samuel ER, Lowdell MW. Manufacturing of highly functional and specific T cells for adoptive immunotherapy against virus from granulocyte colony-stimulating factor-mobilized donors. Cytotherapy 2014.

19. Rutella S, Pierelli L, Bonanno G, Sica S, Ameglio F, Capoluongo E, et al. Role for granulocyte colony-stimulating factor in the generation of human $T$ regulatory type 1 cells. Blood. 2002;100:2562-71.

20. Bunse CE, Borchers S, Varanasi PR, Tischer S, Figueiredo C, Immenschuh S, et al. Impaired functionality of antiviral T Cells in G-CSF mobilized stem cell donors: implications for the selection of CTL donor. PLoS One. 2013;8:e77925

21. Tanel A, Fonseca SG, Yassine-Diab B, Bordi R, Zeidan J, Shi Y, et al. Cellular and molecular mechanisms of memory T-cell survival. Expert Rev Vaccines. 2009;8:299-312.

22. Surh CD, Boyman O, Purton JF, Sprent J. Homeostasis of memory T cells. Immunol Rev. 2006;211:154-63.

23. Swain SL, McKinstry KK, Strutt TM. Expanding roles for CD4(+) T cells in immunity to viruses. Nat Rev Immunol. 2012;12:136-48.

24. Bracci L, Moschella F, Sestili P, La Sorsa V, Valentini M, Canini I, et al. Cyclophosphamide enhances the antitumor efficacy of adoptively transferred immune cells through the induction of cytokine expression, B-cell and T-cell homeostatic proliferation, and specific tumor infiltration. Clin Cancer Res. 2007;13:644-53.

25. Muranski $P$, Boni A, Wrzesinski C, Citrin DE, Rosenberg SA, Childs R, et al. Increased intensity lymphodepletion and adoptive immunotherapy-how far can we go? Nat Clin Pract Oncol. 2006;3:668-81.

26. Focosi D, Bestagno M, Burrone O, Petrini M. CD57+ T lymphocytes and functional immune deficiency. J Leukoc Biol. 2010;87:107-16.

27. Brenchley JM, Karandikar NJ, Betts MR, Ambrozak DR, Hill BJ, Crotty LE, et al. Expression of CD57 defines replicative senescence and antigen-induced apoptotic death of CD8+ T cells. Blood. 2003;101:2711-20.
28. Scheinberg P, Melenhorst JJ, Brenchley JM, Hill BJ, Hensel NF, Chattopadhyay PK, et al. The transfer of adaptive immunity to CMV during hematopoietic stem cell transplantation is dependent on the specificity and phenotype of CMV-specific T cells in the donor. Blood. 2009;114:5071-80.

29. Sallusto F, Lenig D, Forster R, Lipp M, Lanzavecchia A. Two subsets of memory $T$ lymphocytes with distinct homing potentials and effector functions. Nature. 1999;401:708-12.

30. Maus MV, Fraietta JA, Levine BL, Kalos M, Zhao Y, June CH. Adoptive immunotherapy for cancer or viruses. Annu Rev Immunol. 2014;32:189-225.

31. Lee SA, Sinclair E, Hatano H, Hsue PY, Epling L, Hecht FM, et al. Impact of HIV on CD8+ T cell CD57 expression is distinct from that of CMV and aging. PLoS One. 2014;9:e89444

32. Betjes MG, Meijers RW, de Wit EA, Weimar W, Litjens NH. Terminally differentiated CD8+ Temra cells are associated with the risk for acute kidney allograft rejection. Transplantation. 2012;94:63-9.

33. Pera A, Campos C, Corona A, Sanchez-Correa B, Tarazona R, Larbi A, et al. CMV latent infection improves CD8+ T response to SEB due to expansion of polyfunctional CD57+ cells in young individuals. PLoS One. 2014;9:e88538.

34. Le Priol Y, Puthier D, Lecureuil C, Combadiere C, Debre P, Nguyen C, et al. High cytotoxic and specific migratory potencies of senescent CD8+ CD57+ cells in HIV-infected and uninfected individuals. J Immunol. 2006;177:5145-54.

35. Lachmann R, Bajwa M, Vita S, Smith H, Cheek E, Akbar A, et al.

Polyfunctional T cells accumulate in large human cytomegalovirus-specific T cell responses. J Virol. 2012;86:1001-9.

36. Hasan MS, Kallas EG, Thomas EK, Looney J, Campbell M, Evans TG. Effects of interleukin-15 on in vitro human T cell proliferation and activation. J Interferon Cytokine Res. 2000;20:119-23.

37. Ewen $\mathrm{CL}$, Kane KP, Bleackley RC. A quarter century of granzymes. Cell Death Differ. 2012;19:28-35.

38. Sallusto F, Geginat J, Lanzavecchia A. Central memory and effector memory $T$ cell subsets: function, generation, and maintenance. Annu Rev Immunol. 2004;22:745-63.

39. Uhlin M, Gertow J, Uzunel M, Okas M, Berglund S, Watz E, et al. Rapid salvage treatment with virus-specific $T$ cells for therapy-resistant disease. Clin Infect Dis. 2012:55:1064-73.

40. van Loenen MM, de Boer R, van Liempt E, Meij P, Jedema I, Falkenburg JH, et al. A Good Manufacturing Practice procedure to engineer donor virus-specific T-cells into potent anti-leukemic effector cells. Haematologica. 2013.

\section{Submit your next manuscript to BioMed Central and take full advantage of:}

- Convenient online submission

- Thorough peer review

- No space constraints or color figure charges

- Immediate publication on acceptance

- Inclusion in PubMed, CAS, Scopus and Google Scholar

- Research which is freely available for redistribution

Submit your manuscript at www.biomedcentral.com/submit 